Narváez Cardona, E., y Zúñiga Castillo, R. (mayo-agosto, 2021). Explorar la escritura y la comunicación profesional: casos de publicidad social para ONG. Revista Virtual Universidad

Católica del Norte, (63), 245-276. https://www.doi.org/10.35575/rvucn.n63a10

\title{
Explorar la escritura y la comunicación profesional: casos de publicidad social para ONG
}

Exploring professional writing and communication: cases of Social Advertising in NGO

\author{
Elizabeth Narváez Cardona \\ Doctora en Educación \\ Departamento de Educación, Universidad Autónoma de Occidente \\ Cali, Colombia \\ enarvaez@uao.edu.co
}

Orcid: https://orcid.org/0000-0002-4589-8331

CvLac:

http://scienti.colciencias.gov.co:8081/cvlac/visualizador/generarCurriculoCv.do?cod_rh=0000257214

\section{Rodrigo Zúñiga Castillo}

Publicista, Joven Investigador

Departamento de Educación, Universidad Autónoma de Occidente

Cali, Colombia

rodrigozunigac1@gmail.com

Orcid: https://orcid.org/0000-0001-8452-4589

CvLac: https://scienti.minciencias.gov.co/cvlac/visualizador/generarCurriculoCv.do?cod rh=0001840339

Recibido: 1 de julio de 2020

Evaluado: 12 de noviembre de 2020

Aprobado: 26 de febrero de 2021

Tipo de artículo: Investigación Científica y Tecnológica

\section{Resumen}

Se documentaron experiencias profesionales de publicidad social para ONG, desde perspectivas sistémicas de la escritura y la comunicación profesional. Estudios sobre la escritura profesional han documentado la importancia de incluir perspectivas sistémicas que visibilicen cómo viajan las producciones escritas y entre quiénes. Los enfoques sistémicos exploran el comportamiento 
humano colectivo en el marco de interacciones cooperativas y competitivas, en las que hay una división social del trabajo, así como acceso jerárquico a recursos, derechos y deberes. Con dicho enfoque, se desarrolló un estudio basado en entrevistas estructuradas a ocho profesionales de la comunicación y la publicidad. El protocolo de entrevista constó de 21 preguntas sobre: 1) información de la organización, 2) perfil y trayectoria profesional, 3) descripción de un proyecto profesional desafiante, y 4) descripción de la ruta de procedimientos para producir un entregable desafiante. Los análisis confirman que la escritura y la comunicación profesional en publicidad social tienen interacciones con la educación; es decir, el uso de la comunicación como medio para promover el cambio. También, se observa que llevar a cabo proyectos profesionales en ONG tiene lugar en una ecología de entregables, vistos como productos intermedios (bocetos, prototipos o borradores) y finales (manuales de campaña, sitios web, murales). Los resultados pueden usarse para diseñar experiencias de aprendizaje con casos profesionales de simulación, como los reportados en este estudio.

Palabras clave: Comunicación profesional; Escritura profesional; Formación de escritores profesionales; Publicidad social en ONG.

\begin{abstract}
This study explores professional experiences on Social Advertising in NGO from systemic approaches of professional writing and communication. Studies on professional writing have documented the importance of incorporating systemic perspectives to analyze how contents navigate among stakeholders. Systemic approaches allow exploring collective human activities addressing a goal through cooperation and competitive interactions by social division of labor, and hierarchical access to resources, rights, and duties. Within this framework, a study was conducted through eight structured interviews applied by a 21-question protocol regarding: 1) organization's information; 2) professional profile and trajectory; 3) description of a challenging professional project; and, 4) description of procedures for producing a challenging deliverable. The analysis confirms that professional writing and communication on Social Advertising in NGO interacts with education (i.e., communication as means for change). Additionally, undertaking professional projects in NGO takes place in a deliverables' ecology of provisional (sketches, prototypes or
\end{abstract}


drafts), and final products (campaign manuals, websites, murals). The results might be utilized to offer learning experiences by incorporating professional simulated cases, as those reported by this study.

Keywords: Professional communication; Professional writing; Professional writers education; Social advertising in NGOs.

\section{Introducción}

Estudios de la escritura especializada en América Latina abogan por intervenciones pedagógicas, con orientación disciplinar y profesional ${ }^{1}$ (Carlino, 2013; Navarro et al., 2016; Parodi y Burdiles, 2015); este movimiento pedagógico ha sido permeado por enfoques estadounidenses, conocidos como escritura en las disciplinas y profesiones (Writing in the disciplines, WID, por sus siglas en inglés). Dicha perspectiva defiende el diseño curricular de experiencias de aprendizaje en los campos asociados a la formación disciplinar y profesional (Russell, 2007) y cada vez se ha convertido en foco de interés explorar lo que sucede con la lectura y la escritura en el mundo universitario (Islas Torres y Carranza Alcántar, 2020; González Palacio et al., 2021). En América Latina, la tendencia ha sido el uso de la lingüística de corpus para aportar al estudio de la escritura disciplinar y profesional, y los patrones textuales identificados se privilegian como contenidos de enseñanza para el currículo de la escritura especializada (Navarro et al., 2016).

Sin embargo, investigaciones desarrolladas a partir de observaciones naturalistas de la escritura y la comunicación profesional han documentado la importancia de incluir perspectivas sistémicas que visibilicen cómo viajan las producciones escritas y entre quiénes (Spinuzzi, 2014; Winsor, 2013). En estos enfoques, los contenidos de las disciplinas y profesiones se generan a partir de un entramado de actuaciones que incluyen conversación, interacción e interdependencia entre varios participantes, y que se concretan en productos finales. Estas perspectivas sistémicas

\footnotetext{
${ }^{1}$ En este estudio se consideran diferencias entre las disciplinas y profesiones, con base en las distinciones entre prácticas académicas y científicas, propuestas por Russell \& Cortes (2012), quienes afirman que la mayoría de estudiantes universitarios no pasan a convertirse en investigadores, sino en profesionales de instituciones no académicas, en las que se demanda una escritura profesional, pero no científica. Se asume, en consecuencia, que las disciplinas tienen una orientación científica más que las profesiones, aunque las dos prácticas, académicas y científicas, pueden considerarse partes funcionales de interacciones en contextos políticos y económicos más amplios (ej., utilizar la investigación para la toma de decisiones profesionales).
} 
pueden ser útiles para concebir la escritura no solo como un proceso de alta demanda intelectual, generalmente vivido en solitario, sino como un proceso comunicativo; es decir, la escritura como fenómeno interactivo socialmente organizado y asimétrico entre participantes, que se apoya en diversas materialidades, además de las alfabéticas.

Para el caso de la escritura especializada en el campo de la publicidad, se observa que los estudios tienden a caracterizar y analizar la producción del texto escrito conocido como el copy (Aldás, 2007; Castellblanque, 2009; Lahoz, 2007). Por eso, tal vez, los currículos en publicidad incorporan la enseñanza del copy como parte central de la formación. La revisión de planes de estudio de 13 universidades en Colombia, que obtuvieron los mejores resultados en 2017 en una evaluación masiva nacional y obligatoria de la calidad de la educación superior, muestra que 10 programas (77\%), incluyen asignaturas asociadas a la enseñanza del copy.

Por otro lado, la revisión de publicaciones sobre escritura en publicidad muestra, además, que los trabajos presentan un énfasis en el análisis técnico de los entregables y su recepción por parte de los usuarios finales (Castellblanque, 2009; Huertas-García et al., 2014; Lahoz, 2007). Este contexto podría sugerir que la formación de los publicistas exige la reflexión de cómo se produce el copy, pero también incluir el estudio de otros procesos de diseño y recepción de entregables.

Por consiguiente, enfoques sistémicos sobre la escritura y la comunicación ayudan a entender la actuación profesional en el campo de la publicidad, más allá de la producción del copy. Este estudio describe actuaciones profesionales para el campo de la comunicación en ONG, porque se asumió que en ese contexto emergerían estrategias de generación de contenidos, además de la creación de copy. También se puede entender el copy, copywrite o redacción publicitaria, como la aplicación de técnicas de redacción en publicidad que busca la corrección, adaptación y eficacia del mensaje escrito (Gutiérrez Guerrero y Moscardó Herrera, 2012).

El estudio responde las siguientes preguntas: a) ¿Qué caracteriza la comunicación y la escritura en publicidad social de ONG, en casos documentados en el suroccidente de Colombia?; y b) ¿Cómo se producen contenidos en los que se involucra un publicista que trabaja en una ONG en dicho contexto regional? Los resultados de este estudio pueden usarse para nutrir decisiones curriculares que orienten la formación del publicista, que vayan más allá de contenidos textuales del copy e incluyan experiencias de aprendizaje con casos profesionales de simulación, como los reportados en este trabajo. 


\section{Revisión de literatura}

Este estudio adopta el marco de referencia de las comunidades de práctica (Blackmore, 2010; Wenger, 2010), el cual explica que los seres humanos aprenden como parte de la acción colectiva y, por eso, la transformación humana, su evolución y consecución de experticia/competencia es solo posible en contextos de participaciones grupales en las que se gana membresía y se desarrollan identidades de afiliación con uno o varios grupos de constitución institucional formal (ej., una empresa, un colectivo de arte) o informal (ej., la familia, el género, la profesión). Bajo este enfoque, la acción humana y el aprendizaje en sistemas de relaciones de interdependencia $y$, por consiguiente, la experticia o la competencia no se concibe como un conjunto de saberes y habilidades que van a ser adquiridos en su totalidad por un solo individuo en un momento específico, sino que se trata de saberes y habilidades que podrán estar dispersos, articulados y bajo el dominio de un grupo, dependiendo de las condiciones en las que se desarrolle la actividad colectiva y las metas que se persigan (Blackmore, 2010; Chaiklin y Lave, 2001; Spinuzzi, 2015; Wenger, 2010).

Esta perspectiva sistémica del aprendizaje y la acción social se complementa con el modelo teórico de la teoría de la actividad. Este enfoque explora el comportamiento humano colectivo dirigido hacia un objetivo, situado históricamente en el marco de interacciones humanas cooperativas y, al mismo tiempo, competitivas, en las que hay una división social del trabajo, debido al acceso jerárquico a los recursos y herramientas disponibles, tanto materiales como simbólicas, lo que deriva en variedad de derechos y deberes, para y entre los participantes (Engeström \& Sannino, 2016; Spinuzzi, 2015).

Este estudio retoma las contribuciones de la teoría de la actividad como una lente para describir las acciones humanas, especialmente el desempeño profesional basado en resultados; en dicho marco, el lenguaje se convierte simultáneamente en herramienta mediadora y productos finales que van tejiendo y son tejidos por la actividad humana colectiva. Este fenómeno está estructurado por roles, jerarquías y contradicciones, debido a la superposición de motivos personales y objetivos colectivos; tensiones que ofrecen oportunidades para la transformación individual o grupal (Engeström, 2001, 2014; Spinuzzi, 2015). 
Publicaciones asociadas con la escritura profesional de publicidad, redacción en publicidad y discurso publicitario2 muestran que los trabajos se centran en temáticas como los efectos de la marca y el producto, a partir del eslogan publicitario (Gutiérrez Guerrero y Moscardó Herrera, 2012); también, en el estudio de las figuras retóricas desde el análisis de la pragmática, la retórica aristotélica y otras corrientes argumentativas (Fazio, 2009). Desde enfoques similares, Hernández (2010) trabaja sobre el uso de los recursos literarios en la publicidad, a partir de la retórica, la poesía y los géneros literarios. Por otro lado, Akinbode (2012) explora textos publicitarios desde un análisis sociolingüístico (sintaxis y semántica). Toledo (2007), por su parte, hace una propuesta de análisis del género discursivo, más allá del texto, al asumir "el contexto" como el proceso de recepción de los anuncios. Otra temática explorada en los trabajos es el uso de extranjerismos en la escritura del mensaje comercial (Ávila, 2014).

En cuanto al estudio del mensaje publicitario, se afirma que se estudia como acto discursivo (Riaño, 2016), desde los postulados de la semiótica de Charles Sanders Peirce y su posible proyección a la comunicación contemporánea, para proponer un modelo de planeación estratégica (Strategic Planning). En este trabajo se ejecuta un proyecto piloto con un cliente real para evaluar la viabilidad de la planeación propuesta.

También, se encuentran trabajos donde se revisa cómo se aprovechan los diferentes elementos de los lenguajes televisivos y radiofónicos en la publicidad para describir los diferentes tipos de relación que se pueden establecer entre cuñas y spots de una misma campaña, en función del grado de similitud o fidelidad entre los contenidos (i Suñé y Foix, 2011).

Rey (2011) presenta un trabajo centrado en la evolución del texto publicitario, al documentar cambios en la redacción de los anuncios durante un período de cien años; destaca la transformación del lector como consumidor y una simplificación de la sintaxis. Finalmente, el trabajo de Riaño (2016) defiende la publicidad como agente cultural que hace posible la expresión de tendencias estéticas, sociales-culturales, políticas e ideológicas, e incluso es agente educativo. El autor enfatiza en la necesidad de centrar la atención en los efectos prácticos de las acciones

\footnotetext{
${ }^{2}$ Se realizaron búsquedas en español de trabajos empíricos bajo los siguientes términos clave: Escritura en publicidad, lectura publicitara, el copy, redacción publicitaria y discurso publicitario. Las búsquedas se realizaron desde septiembre de 2016 hasta octubre de 2017. Los lugares de indagación fueron: Google académico, Redalyc y Dialnet, y las fechas de los documentos revisados estuvieron entre 2007 y 2017. Se revisaron un total de nueve documentos.
} 
tácticas publicitarias para relacionar el efecto del mensaje publicitario y su relación con directrices de planeación estratégica.

La revisión exploratoria de literatura sobre estos trabajos en el campo de la publicidad muestra una concentración de estudios sobre la construcción (gramatical, discursiva, literaria y/o retórica) del mensaje publicitario, su circulación e impacto, más que en la exploración de la actuación profesional de los publicistas en el contexto de experiencias colectivas, que es el marco teórico que se integra en el presente estudio.

En cuanto a las relaciones entre la comunicación, la publicidad social y las ONG, la revisión de literatura sugiere que la publicidad social puede verse como una dimensión estratégica del marketing social, que surgió en el contexto estadounidense como una manera de articular las expectativas de la industria y la academia para mostrar sensibilidad hacia temas sociales. El marketing social se entiende como la aplicación de técnicas de marketing, en un sentido comercial, que tienen el propósito de generar un bien social, ya sea incentivando la compra de un producto o un cambio de comportamiento (Friedman et al., 2016). El uso de la publicidad como estrategia central en campañas de marketing social se ha potencializado por el uso de tecnologías digitales para la difusión de contenidos (Firestone et al., 2017). En consecuencia, las técnicas usadas por el mercadeo comercial han servido de plataforma para el marketing social, dado que, en últimas, tanto el mercadeo como la publicidad social buscan modificar actitudes y comportamientos en las personas (Gumucio-Dragón, 2011; Kubacki \& Szablewska, 2019).

La comunicación, como actividad profesional, por su parte, es también una práctica que puede estar asociada al mercadeo y a la publicidad social. Dicha actividad profesional se destaca como un mecanismo utilizado por las ONG para dar a conocer sus principios y acciones, aunque no necesariamente tengan objetivos de marketing social (Arroyo Almaraz, Baladrón Pazos et al., 2013).

La comunicación que aprovecha estrategias de social media, que incluye todas las plataformas digitales de comunicación que permiten la realización e interacción de contenido por parte de los usuarios (Gutiérrez Guerrero y Moscardó Herrera, 2012), apoya la creación de conciencia y el fomento de la opinión pública, a través de la comunicación participativa, la generación de movimientos sociales y un sentimiento de compromiso ciudadano (Pawlak, 2020; Senes y Ricciulli-Duarte, 2019). Por otro lado, la gestión de la comunicación en las ONG se 
desarrolla de manera integral, aunque se haya concentrado inicialmente en áreas de prensa. Por ejemplo, se han ido incorporando estrategias de comunicación interna y externa, como el desarrollo de campañas publicitarias para consecución de financiación (González et al., 2016).

Las ONG de desarrollo, agencias orientadas al cambio social, buscan ofrecer información sobre sus proyectos, sensibilizar sobre las causas sociales que defienden, captar voluntariado y obtener recursos. Se afirma que el $60 \%$ de las personas contratadas para el desarrollo de actividades de comunicación, también realizan otras actividades como manejo de archivo o la coordinación del voluntariado (Nieto, 2007). Al parecer, el $50 \%$ de los responsables de las comunicaciones de las ONG no son profesionales de la comunicación, por lo cual se afirma que, en ocasiones, este tipo de organizaciones producen contenidos ineficientes (Göttlichová \& Soukalová, 2015; Nieto, 2007).).

Esto indicaría que las ONG necesitarían de profesionales de la comunicación para aportar en la identidad organizacional, las alianzas con organizaciones públicas o privadas y la priorización de los grupos de interés (Liston-Heyes \& Liu, 2013), a través de, idealmente, campañas de publicidad social. Sin embargo, la creación e implementación de una campaña de publicidad social requiere de saberes especializados, de los que el voluntariado no necesariamente dispone, como estrategias investigativas y diseño de programas por medio de metas y objetivos (González-Cabrera, 2017; Toca Torres, 2013).

La revisión realizada coincide en que la escasez de presupuesto afecta los proyectos de publicidad y comunicación en las ONG y limita las posibilidades de comunicar en diversos medios. Se dice que se requiere de estrategias de comunicación cada vez más responsables al impactar al público al que se dirigen. Ante estas exigencias, la innovación y aplicación de tecnologías digitales son vistas como una alternativa para la realización de publicidad social (Almaraz, 2014; Almaraz et al., 2013; Göttlichová \& Soukalová, 2015; López, 2009).

En relación con el contexto colombiano, un análisis exploratorio de contenido de 73 noticias online, disponibles en 2018 en los dos periódicos más antiguos de Colombia, El Espectador y El Tiempo, asociadas a ONG en el contexto político y social colombiano, muestra que las ONG se concentran en temas de violencia, derechos humanos y líderes sociales (20), el conflicto armado (18), y cambios en la política pública (11). En menor presencia, se ocupan de 
temas sobre participación ciudadana (5), iniciativas medioambientales (5), la salud (4), apoyo y desarrollo económico (4), tecnología (3) y narcotráfico (3).

\section{Metodología}

El estudio fue descriptivo y basado en participación voluntaria de los informantes; por eso los resultados obtenidos solo describen tendencias entre los conteos y no se utilizan con fines de generalización (Bonilla-García y López-Suárez, 2016; Merriam, 1998; Schettini y Cortazzo, 2015).

\section{Fase 1: Reclutamiento de participantes}

Se elaboraron, registraron en video y analizaron entrevistas estructuradas a ocho profesionales de la comunicación, que trabajaban o habían realizado actividades de comunicación para ONG. La búsqueda y selección de los profesionales se realizó a través de: 1) base de datos de egresados de la universidad en la que se inscribió el proyecto; 2) base de datos de una encuesta aplicada en el proyecto marco al que se asociaba este estudio ${ }^{3}$; y 3) directorios locales de ONG a través de Google (directorio de agremiaciones y asociaciones de la Corporación Autónoma Regional del Valle del Cauca, y directorio de ONG de la página web de la Alcaldía de Santiago de Cali, Colombia).

La invitación a los entrevistados se realizó por vía telefónica y correo electrónico, adjuntando el consentimiento informado. Dado que su participación fue voluntaria, el estudio se basó en una muestra no intencional y por conveniencia (Etikan et al., 2016); por eso, finalmente pudieron reconstruirse ocho casos de profesionales que aceptaron participar de manera voluntaria en el estudio.

\footnotetext{
${ }^{3}$ El presente trabajo está adscrito al proyecto "Estudio sobre prácticas de escritura de egresados en organizaciones de trabajo aportando a los resultados de las pruebas de comunicación escrita Saber Pro en Colombia". Dicho estudio incluyó el diseño, pilotaje y aplicación de una encuesta cualitativa digital y de respuesta abierta aplicada a egresados de los campos objeto de análisis (comunicación social - periodismo, publicidad, diseño gráfico, ciencias ambientales, salud y educación), para describir prácticas de escritura en el contexto laboral.
} 


\section{Fase 2: Diseño y aplicación de un protocolo de entrevista estructurada}

Generar contenidos de comunicación en o para organizaciones implica la coordinación de diversas actividades y participantes. La teoría de la actividad fue en este estudio un modelo teórico y metodológico que, desde perspectivas sistémicas, ayuda a describir los aspectos que influyen en la coordinación de dichas actividades y participantes a lo largo del tiempo. La unidad base de la teoría de la actividad es el "sistema de actividad", que se define como un grupo de personas quienes comparten un objetivo y motivación en común, por un periodo de tiempo, mientras utilizan diversas herramientas que permitan lograr dicho objetivo (Kain \& Wardle, 2014). En este estudio se hicieron visibles sistemas de actividad, al identificar ciclos de flujo de información en la creación de contenidos de comunicación en o para ONG.

Identificar estos ciclos de flujo en la generación de contenidos permitió describir las acciones realizadas para la consecución de un objetivo/producto de comunicación concreto. Esta descripción es una ventana metodológica para comprender qué clase de publicidad realizan los profesionales de comunicación en este tipo de organizaciones.

Por consiguiente, se utilizó como estrategia metodológica, la exploración de experiencias profesionales desafiantes, porque se comprende a las personas como agentes que están siempre en capacidad de aprender, especialmente cuando se enfrentan a experiencias poco rutinarias que les exigen transformación (Engeström \& Sannino, 2010; Rounsaville, 2012; Tuomi-Gröhn et al., 2003). Las experiencias profesionales se documentaron a partir de un protocolo de entrevista estructurada que constó de 21 preguntas, organizadas en las siguientes cuatro secciones: 1) información de la organización, 2) perfil y trayectoria profesional, 3) descripción de un proyecto profesional desafiante, y 4) descripción de la ruta de procedimientos utilizados para producir un entregable desafiante (anexo 1).

Para apoyar el ejercicio retrospectivo, al responder las preguntas de la sección 4, se solicitó a los entrevistados dibujar, mientras hablaban o después de hablar, la ruta de procedimientos utilizados para producir el entregable desafiante. Esta estrategia de recolección de información es una réplica de la técnica utilizada por Paul Prior \& Jody Shipka (2003) que consiste en solicitar un dibujo que represente cómo se llevan a cabo proyectos escriturales: lugares, episodios de escritura, recursos, personas involucradas, circulación de otros textos y sentimientos. Además, se les solicitó 
mencionar: los participantes involucrados, los roles de los participantes en la ruta de producción, los espacios físicos u otros materiales utilizados para la producción y los tiempos de producción.

Estas visualizaciones fueron comparadas con visualizaciones construidas por el analista para crear una cronología de la ruta de procedimientos reportada por los entrevistados. La ruta de procedimientos se entendió como el conjunto de actividades profesionales que pueden situarse en el tiempo y podían repetirse en el proceso de realización de un entregable.

Por ejemplo, la integración de la figura 1 y 2 representa una experiencia profesional en publicidad para una ONG especializada en salud. El objetivo era comunicar sobre tipos de cáncer en la infancia. Para desarrollar el producto de comunicación, el publicista realizó encuentros iterativos con los profesionales médicos encargados de la ONG para entender los contenidos a comunicar y definir cómo adaptarlos a las audiencias. Una vez entendidos los contenidos, se generó la idea de crear una infografía para el sitio web de la ONG. La realización de ese entregable requería del apoyo de un ilustrador, con quien se generaron diversas versiones de la infografía, hasta recibir la aprobación final de los profesionales médicos y directivos de la ONG. Los recuadros en color rojo en ambas figuras ilustran acciones del publicista para comprender los contenidos a comunicar, mientras que los recuadros azules ilustran actividades que son dependientes de otros participantes para su aprobación y realización.

\section{Figura 1}

Dibujo realizado por el entrevistado

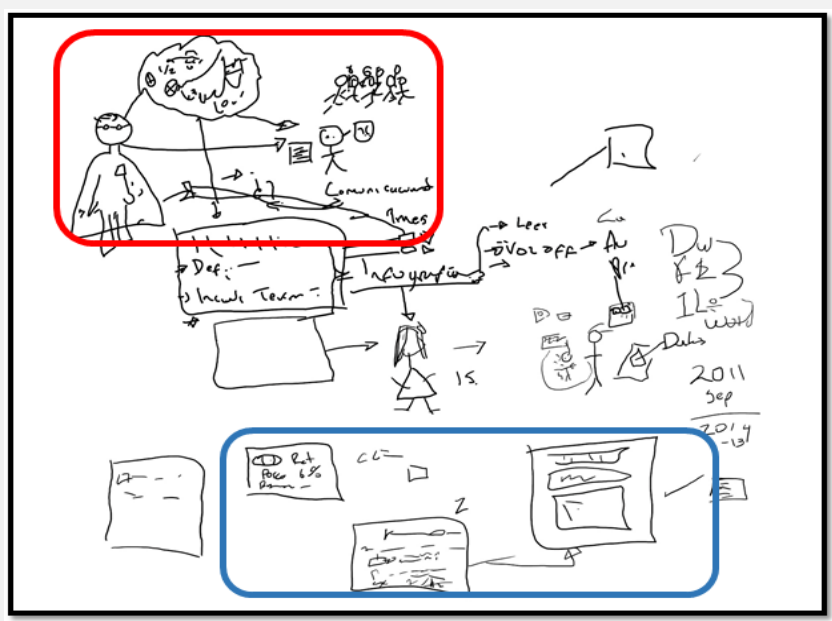

Fuente: elaboración propia. 


\section{Figura 2}

Recreación ilustrada y cronológica del analista

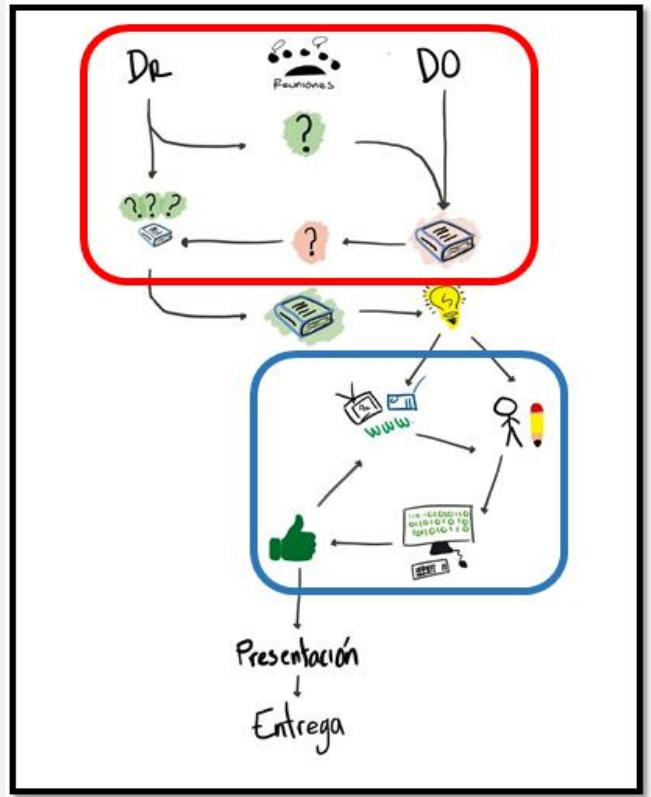

Fuente: elaboración propia.

\section{Fase 3: Procesamiento y análisis de las transcripciones de las entrevistas}

En una tabla de Excel, se realizaron transcripciones ortográficas de las entrevistas y se organizaron por turnos de habla, marcados por el tiempo de producción oral del entrevistador y del entrevistado. Se utilizaron dos niveles de análisis y codificación inductiva. El primer nivel identificaba la ruta de procedimientos utilizados para producir el entregable desafiante en cada uno de los ocho casos. En otra tabla de Excel se describían, de manera separada, los procedimientos identificados por el analista, de aquellos identificados por los entrevistados. La información organizada en dicha tabla de Excel y las ilustraciones realizadas por los entrevistados fueron comparadas para identificar recurrencias entre los casos.

En el segundo nivel se utilizó el software Atlas. Ti versión 8.1., para revisar y corroborar los códigos creados en el primer nivel. Este análisis permitió hacer conteos de presencia de menciones a lo largo de las ocho transcripciones ortográficas de las entrevistas. Posteriormente, se 
crearon grupos de códigos para comprender actuaciones profesionales relacionadas; por ejemplo, rutas de procedimientos asociadas a la producción de contenidos digitales versus análogos, rutas de procedimientos asociados a la creación de entregables intermedios versus entregables finales, y rutas de procedimientos con actuación profesional individual versus actuación profesional interdependiente con otros actores, como jefes, colegas de profesión y colegas de otras profesiones.

\section{Resultados}

La identificación de los proyectos desafiantes y las metas perseguidas en ellos evidenció que las ONG buscaban el reconocimiento de su organización por parte de sus públicos objetivos (casos 5 y 6, en tabla 1). Las metas u objetivos identificados a lo largo de los casos indicaban también que creaban contenidos de comunicación con propósitos educativos. Por ejemplo, buscaban educar sobre estados de salud, creando comunicación que permitiera explicar de una forma más cotidiana las enfermedades, y teniendo en cuenta audiencias de poblaciones vulnerables (casos 2 y 3 , en tabla 1$)$.

Se pudieron identificar diferentes audiencias entre los casos; algunas podían ser amplias, toda una población de una ciudad (caso 1, en tabla 1), o más delimitadas, personas con una enfermedad específica (caso 2, en tabla 1).

En relación con el entregable o producto final, asumiendo el entregable como una unidad materialmente delimitada, se observó que los soportes eran tanto digitales como análogos, que podían ir más allá de la escritura alfabética y tenían distintos propósitos y audiencias. Por ejemplo, se encontraron entregables digitales (caso 3 y 5, en tabla 1), escritura en formatos digitales (caso 4, en tabla 1), audiovisuales (caso 8), o análogos tridimensionales (caso 1 y 2, en tabla 1).

Esta descripción también permitió comprender que la creación de los "entregables finales" respondía a contenidos físicamente delimitados o ser una actividad interactiva. Por ejemplo, se creó un contenedor (caso 1, en tabla 1 y figura 3) dirigido a posibles donantes de tapas plásticas de envases de bebidas gaseosas, así como el manual de la campaña de la que hacía parte el contenedor (caso 1, en tabla 1 y figura 4); el manual estaba dirigido a futuros publicistas que pudieran replicar la campaña. También, hubo entregables cuya evidencia eran materiales utilizados en talleres, como actividad interactiva in-situ (caso 7, en tabla 1). 


\section{Explorar la}

escritura

\section{Figura 3}

Contenedor para donantes de tapas

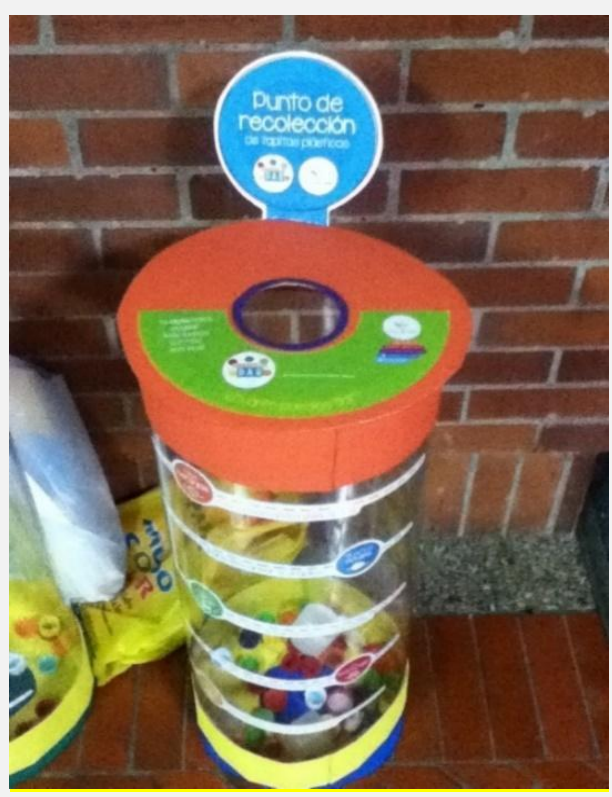

Nota: elaboración propia.

\section{Figura 4}

Manual de campaña para publicistas

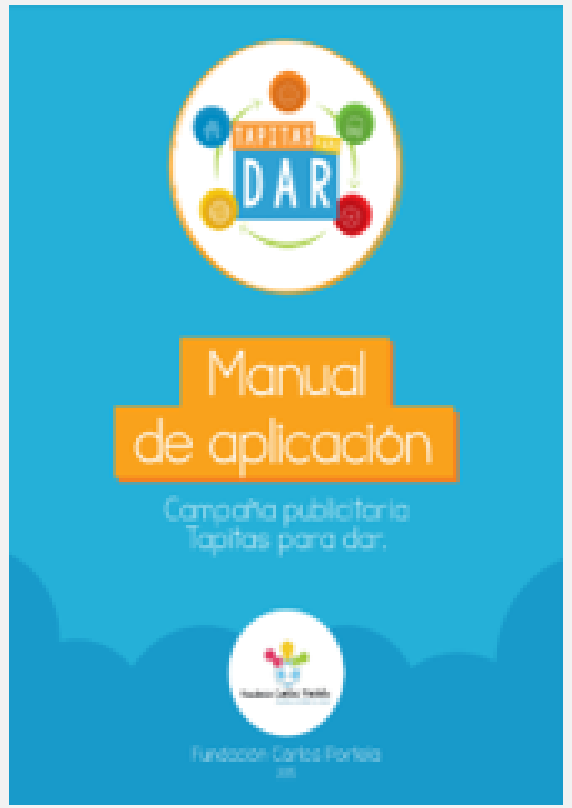

Nota: elaboración propia. 


\section{Tabla 1}

Caracterización de los casos

\begin{tabular}{|c|c|c|c|c|c|c|}
\hline $\begin{array}{l}\text { No. } \\
\text { de } \\
\text { caso }\end{array}$ & $\begin{array}{c}\text { Título } \\
\text { profesional } \\
\text { del } \\
\text { entrevistado }\end{array}$ & $\begin{array}{c}\text { Nombre } \\
\text { del } \\
\text { proyecto }\end{array}$ & $\begin{array}{l}\text { Meta de la } \\
\text { ONG }\end{array}$ & $\begin{array}{l}\text { Objetivo del } \\
\text { proyecto }\end{array}$ & $\begin{array}{l}\text { Nombre del } \\
\text { entregable } \\
\text { final }\end{array}$ & $\begin{array}{c}\text { Posibles } \\
\text { audiencias }\end{array}$ \\
\hline 1 & Publicista & $\begin{array}{l}\text { Tapitas } \\
\text { para dar }\end{array}$ & $\begin{array}{l}\text { Brindar apoyo } \\
\text { integral a } \\
\text { niños con } \\
\text { cáncer }\end{array}$ & $\begin{array}{c}\text { 1) Generar } \\
\text { reconocimiento } \\
\text { de la ONG. 2) } \\
\text { Recaudar fondos } \\
\text { para dar ayuda } \\
\text { financiera al } \\
\text { sostenimiento de } \\
\text { la fundación. 3) } \\
\text { concientización } \\
\text { sobre el } \\
\text { reciclaje. }\end{array}$ & $\begin{array}{l}\text { Contenedor y } \\
\text { manual de } \\
\text { campaña }\end{array}$ & $\begin{array}{c}\text { Donadores, } \\
\text { población } \\
\text { caleña en } \\
\text { general }\end{array}$ \\
\hline 2 & Publicista & $\begin{array}{c}\text { Los } \\
\text { guerrero } \\
\text { s de la } \\
\text { vida }\end{array}$ & $\begin{array}{c}\text { Mejorar las } \\
\text { posibilidades } \\
\text { de curación de } \\
\text { los niños } \\
\text { diagnosticados } \\
\text { con cáncer y } \\
\text { enfermedades } \\
\text { de la sangre en } \\
\text { Colombia }\end{array}$ & $\begin{array}{l}\text { 1) Enseñar a } \\
\text { pacientes sobre } \\
\text { una enfermedad } \\
\text { específica. 2) } \\
\text { Apoyar a } \\
\text { profesionales de } \\
\text { la medicina a } \\
\text { dar explicación } \\
\text { sobre una } \\
\text { enfermedad } \\
\text { específica. }\end{array}$ & Cartilla niños & $\begin{array}{l}\text { Niños, quienes } \\
\text { tienen la } \\
\text { enfermedad } \\
\text { descrita en la } \\
\text { cartilla }\end{array}$ \\
\hline 3 & Publicista & $\begin{array}{c}\text { Diseñar } \\
\text { el sitio } \\
\text { web }\end{array}$ & $\begin{array}{c}\text { Mejorar las } \\
\text { posibilidades } \\
\text { de curación de } \\
\text { los niños } \\
\text { diagnosticados } \\
\text { con cáncer y } \\
\text { enfermedades } \\
\text { de la sangre en } \\
\text { Colombia }\end{array}$ & $\begin{array}{c}\text { Informar sobre } \\
\text { enfermedades de } \\
\text { una manera } \\
\text { didáctica e } \\
\text { intuitiva. }\end{array}$ & $\begin{array}{l}\text { Infografía } \\
\text { multimedia }\end{array}$ & $\begin{array}{l}\text { Niños, padres } \\
\text { de familia, } \\
\text { personas } \\
\text { interesadas en } \\
\text { la enfermedad }\end{array}$ \\
\hline 4 & Publicista & $\begin{array}{c}\text { Evento } \\
\text { Muro al } \\
\text { Barrio }\end{array}$ & $\begin{array}{l}\text { Promoción del } \\
\text { arte en las } \\
\text { comunidades }\end{array}$ & $\begin{array}{l}\text { 1) Resignificar } \\
\text { espacios a partir } \\
\text { del arte. 2) } \\
\text { integrar a la } \\
\text { comunidad en } \\
\text { ambiente sano. }\end{array}$ & $\begin{array}{l}\text { Informe } \\
\text { escrito }\end{array}$ & $\begin{array}{l}\text { La fundación } \\
\text { creadora del } \\
\text { evento, } \\
\text { organizaciones } \\
\text { estatales que } \\
\text { requieran la } \\
\text { información } \\
\end{array}$ \\
\hline
\end{tabular}




\begin{tabular}{|c|c|c|c|c|c|c|}
\hline 5 & $\begin{array}{l}\text { Profesional } \\
\text { en estudios } \\
\text { literarios/ } \\
\text { magíster en } \\
\text { periodismo }\end{array}$ & $\begin{array}{l}\text { Posicion } \\
\text { amiento } \\
\text { del } \\
\text { nombre } \\
\text { de la } \\
\text { organiza } \\
\text { ción }\end{array}$ & $\begin{array}{c}\text { Generar } \\
\text { desarrollo } \\
\text { sostenible para } \\
\text { la zona } \\
\text { pacífica de } \\
\text { Colombia }\end{array}$ & $\begin{array}{c}\text { 1) } \\
\text { Posicionamiento } \\
\text { de la } \\
\text { organización. 2) } \\
\text { Aumentar los } \\
\text { donativos en los } \\
\text { proyectos }\end{array}$ & $\begin{array}{l}\text { Desarrollo de } \\
\text { sitio web }\end{array}$ & $\begin{array}{c}\text { Donadores, } \\
\text { Empresa } \\
\text { privada y } \\
\text { pública que } \\
\text { quiera hacer } \\
\text { donación para } \\
\text { proyectos } \\
\text { sociales. }\end{array}$ \\
\hline 6 & $\begin{array}{c}\text { Comunicador } \\
\text { social- } \\
\text { periodista }\end{array}$ & $\begin{array}{l}\text { Posicion } \\
\text { amiento } \\
\text { de la } \\
\text { organiza } \\
\text { ción }\end{array}$ & $\begin{array}{c}\text { Generar } \\
\text { desarrollo } \\
\text { sostenible para } \\
\text { la zona } \\
\text { pacífica de } \\
\text { Colombia }\end{array}$ & $\begin{array}{c}\text { 1) } \\
\text { Posicionamiento } \\
\text { de la } \\
\text { organización. 2) } \\
\text { Aumentar los } \\
\text { donativos en los } \\
\text { proyectos }\end{array}$ & $\begin{array}{l}\text { Desarrollo de } \\
\text { sitio web }\end{array}$ & $\begin{array}{c}\text { Donadores, } \\
\text { Empresa } \\
\text { privada y } \\
\text { pública que } \\
\text { quiera hacer } \\
\text { donación para } \\
\text { proyectos } \\
\text { sociales. }\end{array}$ \\
\hline 7 & Publicista & $\begin{array}{l}\text { Talleres } \\
\text { de } \\
\text { comunic } \\
\text { ación a } \\
\text { hogares } \\
\text { de paso }\end{array}$ & $\begin{array}{l}\text { Servir de lugar } \\
\text { de paso para } \\
\text { niños en } \\
\text { estado de } \\
\text { vulnerabilidad }\end{array}$ & $\begin{array}{l}\text { 1) Enseñar a } \\
\text { niños de la } \\
\text { fundación las } \\
\text { bases de } \\
\text { lectoescritura }\end{array}$ & $\begin{array}{l}\text { Talleres de } \\
\text { lectura y } \\
\text { escritura, y } \\
\text { sus } \\
\text { materiales }\end{array}$ & $\begin{array}{c}\text { Niños } \\
\text { pertenecientes } \\
\text { a los hogares } \\
\text { de paso } \\
\text { asociados a la } \\
\text { fundación }\end{array}$ \\
\hline 8 & $\begin{array}{c}\text { Comunicador } \\
\text { social- } \\
\text { periodista }\end{array}$ & $\begin{array}{l}\text { Video } \\
\text { de } \\
\text { gestión }\end{array}$ & $\begin{array}{c}\text { Dar } \\
\text { visibilización } \\
\text { al } \\
\text { emprendimient } \\
\text { o social }\end{array}$ & $\begin{array}{l}\text { 1) Invitación a } \\
\text { participar de } \\
\text { evento }\end{array}$ & $\begin{array}{l}\text { Video } \\
\text { comercial }\end{array}$ & $\begin{array}{l}\text { Todos los } \\
\text { interesados en } \\
\text { asistir al } \\
\text { evento }\end{array}$ \\
\hline
\end{tabular}

Nota: elaboración propia.

\section{Procedimientos en la producción de contenidos}

Los proyectos desafiantes se consolidaban a través de la creación de entregables intermedios y finales. Esto significa que había productos, especialmente análogos escritos alfabéticos, que se volvían previos o mediadores del entregable/producto final. Por ejemplo, en el caso 8 del proyecto desafiante "video de gestión", en el que el entregable final era un video comercial, el profesional entrevistado explicaba que, para su producción, realizó un guion escrito y visual": "Yo hice toda la escritura del guion, cómo lo quería en imágenes y cómo quería que se

\footnotetext{
${ }^{4}$ Los fragmentos de las entrevistas se presentarán asociándolos a los casos y marcando el tiempo en minutos y segundos de la transcripción.
} 
escuchara la voz, y el editor editaba lo que le pasaba de imágenes y textos, en congruencia, y sacar finalmente el video" (Caso 8: 32:50-33:32).

Para la creación de dichos entregables, tanto intermedios como finales, se identificaron 31 procedimientos. Dichos procedimientos se determinaron a partir de códigos creados por el analista, que ayudaron a identificar las actividades profesionales que podrían ser recurrentes en el proyecto desafiante descrito por los participantes y que implicaban la interacción con otros para crear contenidos específicos o tomar decisiones relacionadas con los mismos. Los siguientes códigos, con conteos por encima de ocho (que era la cantidad de los entrevistados), se consideraron visibles en la actividad profesional, relacionada con la comunicación y la publicidad en ONG: 1) validación de producto/concepto (21 menciones), 2) producción de contenidos específicos (17 menciones), 3) contextualización e identificación de necesidades (14 menciones), 4) control de cambios de la propuesta del producto/entregable (11 menciones), 5) creación de comunicación para diferentes actores (10 menciones), 6) delegación de trabajo según experticia (8 menciones), y 7) conceptualización (8 menciones).

El procedimiento "La validación de producto/concepto" fueron actividades relacionadas con la revisión y aprobación de diferentes aspectos con el cliente/jefe para poder continuar con el desarrollo parcial o final de un producto/concepto. Por ejemplo, en el caso 6, con el proyecto "Posicionamiento de la organización", cuyo producto/entregable final fue el desarrollo de un sitio web, el profesional entrevistado mencionaba que: "La producción de contenidos generados por el área de comunicación son validados por el jefe del área, al igual que los diferentes proyectos de las demás áreas, son controlados por el área de comunicación” (Caso 6: 24:25-25:10).

El procedimiento "La producción de contenidos específicos", se refería a las actividades relacionadas con la producción de contenidos digitales o análogos que aportaban a la consecución de la meta final, asociada al proyecto desafiante. En el caso 3, con el proyecto "Diseño de página web", cuyo entregable final fue una infografía multimedia, el profesional entrevistado mencionaba que (refiriéndose a crear una introducción a la infografía multimedia): "Ante la posibilidad de que el público objetivo no entendiera la mecánica de la infografía multimedia, realizaron una introducción, asegurando que su público se ubicará en el funcionamiento del producto" (Caso 3: $55: 25-55: 54)$. 
El procedimiento "La contextualización e identificación de necesidades" se trataba de actividades en las que los profesionales de comunicación debían explorar información que diera pertinencia a los entregables finales o a los proyectos mismos, pensando en los usuarios finales o público objetivo. El caso 4, del proyecto "Evento Muro al Barrio", incluía experiencias de práctica profesional de estudiantes de publicidad en una ONG que generaran estrategias de comunicación, orientadas a poblaciones vulnerables. El profesional entrevistado comentaba que:

Jóvenes de 18, 19 años [refiriéndose a los estudiantes en práctica profesional] van al barrio [nombre del barrio] y se encuentran por lo menos con personas que llevan todas su vida habitando en la calle, en el barrio; en el barrio hay una chatarrería que es centro de expendio de drogas; entonces, los jóvenes [refiriéndose a los estudiantes en práctica profesional], pues, han vivido la experiencia de ir y hablar con las personas que están ahí, con los niños que son drogadictos desde muy temprana edad. (Caso 4: 24:24-25:25)

De otro lado, el procedimiento "El control de cambios de la propuesta del producto/entregable" se asoció al control interno entre los profesionales de comunicación para el desarrollo de un proyecto/producto, antes o después de ser validado por un jefe/cliente. Por ejemplo, en el caso 5, el proyecto "Posicionamiento del nombre de la organización", en el que el entregable final fue el desarrollo de un sitio web, el profesional entrevistado afirmaba que:

Lo que son noticias, lo que son los proyectos y todo, todo lo desarrollamos [refiriéndose al área de comunicaciones], o sea, los mismos productos que tú vas a ver para impresión digital, mucha de esa información es la misma que está colgada en la web, o sea, queremos que toda nuestra comunicación sea en un solo tono, en una misma línea y que la misma información que encuentres en web sea la misma que se entregue en reuniones. (Caso 5: 30:40-21:13)

En el caso de "la creación de comunicación para diferentes actores", se trataba el cómo los profesionales en comunicación gestionaban la comunicación con jefe/clientes para avanzar con los proyectos/entregables, a través de productos intermedios que agilizaran la toma de decisiones. En el caso 2, con el proyecto "Los guerreros de la vida", que entregaba como producto final una cartilla para niños, el entrevistado explicaba que era mejor presentar, al jefe/cliente, la información de forma escrita y presencial, que escrita digital: 
Al doctor [refiriéndose al personal médico que era jefe/cliente de los proyectos] le pasamos [todo por] escrito porque pues ha sido un proceso de entender con el tiempo que ellos no leen [correos electrónicos]. Entonces si le mandamos mucha información, no la lee y al final no nos dice nada, o nos contesta lo que no es. (Caso 2: 44:06 - 44:57)

El procedimiento "La delegación de trabajo según experticia" se trataba de actividades en las que la responsabilidad era asumida por otro profesional, colega o miembro del grupo, porque se dividían las labores según los conocimientos individuales. En el caso 2, con el proyecto "Los guerreros de la vida", cuyo producto final fue una cartilla para niños, se mencionaba en la entrevista, respecto a la finalización del entregable:

La arte-finalización [refiriéndose a los últimos pasos en el desarrollo de la cartilla] es convertir eso en una cartilla con las márgenes, los acabados, especificar todo lo que es el CMYK [modelo de impresión a color], que las fuentes estén en curvas. Bueno, todo para que la cartilla no se vaya a dañar y compaginarlo. Compaginarlo es que las primeras [páginas] van con las últimas, así, tipo cartilla; y en este proceso (...) que tiene unas implicaciones de costo y nosotros no somos los genios en esto, esto sí es algo ya que en la formación se lo dan a un diseñador; entonces, nosotros tenemos otro diseñador que ya tiene un enfoque en la parte de impresos. Entonces, nosotros le pasamos la cartilla y él nos artefinaliza. (Caso 2: 56:52 - 57:53)

Finalmente, el procedimiento denominado "La conceptualización" agrupaba actividades relacionadas con el proceso de crear las ideas que se concretarían en campañas/productos/proyectos. En el caso 3, asociado al diseño de un sitio web, cuyo entregable final fue una infografía multimedia, el profesional entrevistado describía cómo se graficaban ideas y el concepto se desarrolló, a través de diferentes reuniones entre compañeros y jefes:

Esta va a ser la idea en el papel [decía el entrevistado mientras simulaba que escribía en una hoja]. Entonces, en papel, este es el dibujo que va a estar impreso, de aquí se va a desprender este cuadrito que va a decir esto. Acá ya el PDF. Entonces cuando ya hicimos ese proceso, el Doctor nos dice: "Sí, no, me parece bien, hágale”. Igual lo que les decía en un comienzo, la Fundación tiene algo muy bueno, y es que desde el punto de vista de comunicación ellos han dejado que el profesional en el área lo haga. Respeta totalmente. (Caso 3: 52:26-53:26). 


\section{Caracterización de la comunicación y la escritura en publicidad social en las ONG}

La actividad profesional documentada cobró vida en interacción con audiencias profesionales, no profesionales, personas del equipo de trabajo y otros participantes. El análisis cualitativo de los procedimientos permitió identificar cuatro tipos de interacciones: interacciones interdisciplinarias con profesionales de la comunicación, interacciones con diversos públicos de interés, interacción con otros profesionales que no son de la comunicación, e interacción con actores no profesionales.

Para el caso de las interacciones interdisciplinarias con profesionales, se observó la interdependencia con diseñadores gráficos: "primero se lo presentamos a un diseñador, porque él también nos daba ideas de cómo manejar a nivel visual o qué cosas podríamos hacer para la construcción del personaje" (Caso 2: 43:28-43:44).

En cuanto a las interacciones con diversos públicos de interés, en el proyecto desafiante "desarrollo de página web", el entrevistado explicaba que:

Ante la academia y ante diversos actores de que vean que hay una fundación en Cali [refiriéndose a los usuarios finales del sitio web], que está realizando equis proyectos, que está trabajando por el desarrollo de la región y eso nos va posicionando. (Caso 6: 24:2324: 34).

En relación con interacción con profesionales que no son de la comunicación, para el proyecto "Los Guerreros de la Vida", el entrevistado explicaba, al hablar sobre los médicos con quienes trabajaban: "Entonces lo que nosotros tratamos es (...) le mandamos solamente las conclusiones y que él mire y apruebe, o si es algo visual, solo lo visual y ya" (Caso 2: 44:1644:30).

Finalmente, interacciones con actores no profesionales, en el caso del proyecto "Evento Muro al Barrio", el entrevistado decía, al referirse a los integrantes de la ONG:

Te comentaba de cómo una de las experiencias más bonitas, cuando sobre todo los muchachos, pues jóvenes de 18, 19 años [estudiantes en práctica] van al barrio (...) y se encuentran por lo menos con personas que llevan toda su vida habitando en la calle. (Caso 4: 24:30-24:40). 


\section{Discusión}

Este estudio confirma que el perfil ocupacional de un profesional de comunicación para ONG se orienta a la visibilización de la organización, el incremento de la sensibilidad social, la educación de audiencias y la consecución de financiación; por tanto, se ratifica que esta dimensión ocupacional de la publicidad puede ubicarse en el campo del marketing social (Arroyo Almaraz, Baños González et al., 2009; González et al., 2016; Gumucio-Dragón, 2011; Nieto, 2007). Los análisis también muestran que parte del perfil ocupacional se nutre si usa la comunicación para generar, en sus audiencias finales, efectos retóricos con potencial transformativo; por consiguiente, se confirma que esta dimensión ocupacional de la profesión tiene interacciones con la educación, es decir, la publicidad como agente cultural (Pawlak, 2020; Riaño, 2016; Senes y Ricciulli-Duarte, 2019).

Sacar adelante proyectos profesionales en las condiciones ocupacionales que se exploraron tiene lugar en una ecología de entregables, vistos como productos intermedios y finales. Los profesionales entrevistados mencionan contenidos que se materializan en bocetos, prototipos o borradores, que se revisan de manera iterativa con distintos participantes (responsables de distintas áreas de una organización o proyecto) o que se comunican a través de lo que se llamó "entregables intermedios" (correos electrónicos, prototipos en reuniones de validación), para gestionar de manera eficiente la toma de decisiones que viabilice el avance del desarrollo del entregable final. Esta perspectiva sistémica en la producción de contenidos y entregables de la publicidad social, no se encontró en la revisión de literatura sobre estudios de la escritura en publicidad, los cuales se centran más en la exploración de las características sintácticas, semánticas, y retóricas (literarias y persuasivas) de los mensajes publicitarios (Akinbode, 2012; Ávila, 2014; Fazio, 2009; Gutiérrez Guerrero y Moscardó Herrera, 2012; Hernández, 2010; Rey, 2011; Toledo, 2007).

El estudio visibiliza la importancia de la comunicación y la producción de entregables intermedios, que dan viabilidad y tránsito al objeto/meta de la actividad profesional, para lograr llegar al resultado final; esto implicaba, en algunos casos, gestionar de manera eficiente la comunicación con profesionales de otros campos (ej., médicos especialistas con poco tiempo para responder correos electrónicos). En este sentido, el presente estudio aporta una perspectiva en el 
análisis de la actividad profesional en publicidad, asociada a la escritura y la comunicación, que nutre estudios previos como el de Riaño (2016), en el que se exploran los efectos prácticos de las acciones tácticas publicitarias para relacionar el efecto del mensaje publicitario y su relación con la planeación estratégica.

Este trabajo confirma, también, que los profesionales de comunicación en ONG llevan adelante proyectos en medio de cogniciones distribuidas; es decir, gestionando la toma de decisiones, en interacción con distintos participantes (profesionales de su campo, de otros campos o comunidades no profesionales). Estas interacciones son indispensables para definir contenidos y productos que harán parte del entregable final. El tipo de interacción más presente y frecuente en los resultados se da con quienes deben validar los contenidos y que no son necesariamente los usuarios finales. Estos interlocutores son quienes más conocen y auditan que los valores organizacionales que se desean comunicar se materialicen en las propuestas de comunicación. Este proceso que se llamó en el análisis "la validación", al parecer, demanda que los profesionales de la comunicación pongan en relación múltiples expectativas que tendrán que materializar en los entregables, de manera que anticipan contenidos y su diseño desde la perspectiva de las audiencias finales, sino también de los autores/responsables institucionales de los contenidos.

La iteración en el proceso de "validación" y la necesidad de ajustar varias veces, de acuerdo con estas múltiples expectativas, indica que uno de los rasgos que beneficiaría a este tipo de profesionales es una actitud flexible ante la crítica y la disposición frente a la negociación, para poder aceptar, contraproponer y tomar decisiones. Asociado al proceso de validación se encontró que "crear el concepto" para realizar un proyecto/entregable de comunicación requiere múltiples relaciones interactivas, y que llegar al "concepto" es el resultado de una ecología dinámica que tendrá un límite basado, principalmente en las expectativas del jefe/cliente. Los resultados no muestran que el cierre del proceso esté delimitado por cronogramas y presupuestos.

Finalmente, los análisis muestran que estos profesionales trabajan de manera colaborativa para generar contenidos y producir ciertos entregables. Se encontró que solo en el caso 1 la producción total del entregable final fue responsabilidad exclusiva del entrevistado porque se trataba de su trabajo de grado. Esta cognición distribuida, también confirmó que otra de las habilidades que logran desarrollar estos profesionales es la capacidad de delegar y costear saberes 
de otras profesiones, especialmente porque usan distintos materiales, recursos y medios (digital, impreso, análogo gráfico/impreso y tridimensional y talleres).

\section{Conclusiones}

Este estudio documenta la escritura y la comunicación en publicidad social desde perspectivas sistémicas, al explorar experiencias profesionales para el campo de la comunicación en ONG. Los análisis confirman que esta dimensión ocupacional de la profesión tiene interacciones con la educación: el uso de la comunicación como medio para promover el cambio. También, se observa que llevar a cabo proyectos profesionales en ONG tiene lugar en una ecología de entregables, vistos como productos intermedios (bocetos, prototipos o borradores) y finales (manuales de campaña, sitios web, murales).

El presente trabajo podría nutrir decisiones curriculares que orienten la formación en escritura y comunicación profesional, que vayan más allá de contenidos textuales del copy e incluyan experiencias de aprendizaje con casos profesionales de simulación, como los reportados en este estudio. Simulaciones o experiencias de aprendizaje en el marco de proyectos desafiantes, como los descritos en este trabajo, serían una oportunidad para que los estudiantes exploren experiencias laborales basadas en una ecología de producción de entregables intermedios y finales, como parte de proyectos de comunicación en organizaciones como las ONG.

Formar a los profesionales para trabajar por proyectos exige que los planes de estudio ofrezcan experiencias que, como muestran los datos de este estudio, demandan trabajar con diversas comunidades disciplinares, profesionales y otro tipo de colectivos. Los planes de estudio deberían definir de qué manera la formación en proyectos se materializa en los perfiles profesionales y ocupacionales de los egresados, así como el tipo de diseño curricular que aporta a su dominio (tipo de asignaturas -disciplinares, interdisciplinares y proyectos de trabajo en los cursos que potencien el trabajo con distintas disciplinas y comunidades). Dar oportunidades formativas en el currículo, en las que los estudiantes rotan de roles y responsabilidades, podría ser una alternativa importante, en aras de preparar a los futuros profesionales para los contextos, como los documentados con los casos reportados. 


\section{Proyecciones}

Los datos no reportan procesos de validación con los usuarios finales o público objetivo, aspecto que se encontró como una tendencia importante en la revisión de publicaciones sobre escritura en publicidad. Tampoco se observaron procedimientos de análisis de mercado y desarrollo de contenidos basados en investigación, que son saberes especializados, reportados en la revisión de literatura, como necesarios para la creación e implementación de campañas de publicidad social (González-Cabrera, 2017; Toca Torres, 2013); tal vez, porque, como muestra la revisión de literatura, este tipo de proyectos de comunicación se realizan con bajo presupuesto y no se orientan necesariamente a la medición de metas y objetivos, sino a generar viabilidad presupuestal, utilizando tecnologías digitales que reducen costos de operación (Almaraz, 2014; Almaraz et al., 2013 Göttlichová \& Soukalová, 2015; López, 2009). Por consiguiente, se requieren nuevos estudios que documenten la evaluación e impactos de campañas de publicidad social y exploren la complejidad de definir el público objetivo o usuarios finales de los contenidos y productos que harán parte del entregable final, dado que, al parecer, los proyectos se dirigen a múltiples audiencias.

\section{Agradecimientos}

Se agradece a los profesionales que participaron voluntariamente en este estudio y proporcionaron tiempo y materiales para la recopilación y análisis de la información. Este proyecto fue patrocinado por los programas "Jóvenes investigadores" (2017-2019) y "Proyectos de Innovación Educativa" (2017-2019) de la Dirección de Investigaciones y el Centro de Apoyo Académico de la Vicerrectoría Académica de la Universidad Autónoma de Occidente, Colombia. También, se agradece a la profesora y colega del programa de publicidad Ingrid Luengas Fajardo, quien aportó en la revisión de literatura asociada con este trabajo. Finalmente, un agradecimiento a los pares evaluadores, porque sus revisiones ayudaron a comunicar el estudio de mejor manera. 


\section{Referencias}

Akinbode, O. (2012). A sociolinguistic analysis of advertising language in selected Nigerian mass media commercials. Research on Humanities and Social Sciences, 2(8), 26-32. https://core.ac.uk/download/pdf/234673244.pdf

Aldás, E. N. (2007). Lenguaje publicitario y discursos solidarios: Eficacia publicitaria ¿eficacia cultural? (Vol. 260). Icaria Editorial.

Almaraz, I. A. (2014). Publicidad social eficaz desde la universidad: los aspectos experimentales. ZER-Revista de Estudios de Comunicación, 19(37), 191-211. https://addi.ehu.es/bitstream/handle/10810/41184/13538-49618-1PB.pdf?sequence $=1 \&$ is Allowed $=\mathrm{y}$

Almaraz, I. A., González, M. B., y Ors, C. V. W. (2013). Análisis de los mensajes audiovisuales del Tercer Sector en YouTube. Revista Latina de Comunicación Social, (68), 14-27. https://www.doi.org/10.4185/RLCS-2013-980

Arroyo Almaraz, I., Baladrón Pazos, A. J., y Martín Nieto, R. (2013). La comunicación en redes sociales: percepciones y usos de las Ong españolas. Cuadernos.info, (32), 77-88. http://dx.doi.org/10.7764/cdi.32.497

Arroyo Almaraz, I., Baños González, M., y Rodríguez García, T. C. (2009). Publicidad social en las ONG de Córdoba (Argentina). Perfiles de la construcción del mensaje. Revista Latina de Comunicación Social, 12(64), 1011-1029. https://www.doi.org/10.4185/RLCS-642009-877-1.011-1.029

Ávila, S. R. (2014). Lectura y escritura del mensaje comercial: los extranjerismos de los sectores publicitarios de la tecnología de la información y de las comunicaciones. Álabe, (10), 117. https://www.doi.org/10.15645/Alabe.2014.10.7

Blackmore, C. (2010). Social learning systems and communities of practice. Springer.

Bonilla-García, M. Á., y López-Suárez, A. D. (2016). Ejemplificación del proceso metodológico de la teoría fundamentada. Cinta de Moebio, (57), 305-315. https://scielo.conicyt.cl/scielo.php?pid=S0717-554X2016000300006\&script=sci_arttext

Carlino, P. (2013). Alfabetización académica diez años después. Revista Mexicana de Investigación

Educativa,

18(57),

355-381. 
http://www.scielo.org.mx/scielo.php?script=sci_arttext\&pid=S1405$\underline{66662013000200003}$

Castellblanque, M. (2009). Manual del redactor publicitario: ¿reglas, normas, técnicas? ¡rómpelas! ESIC Editorial.

Chaiklin, S., y Lave, J. (Eds.). (2001). Estudiar las prácticas: Perspectivas sobre la actividad y contexto. Amorrortu.

Engeström, Y. (2001). Expansive learning at work: Toward an activity theoretical reconceptualization. Journal of education and work, 14(1), 133-156. https://doi.org/10.1080/13639080020028747

Engeström, Y. (2014). Learning by expanding. Cambridge University Press.

Engeström, Y., \& Sannino, A. (2010). Studies of expansive learning: Foundations, findings and future challenges. Educational Research Review, 5(1), 1-24. https://doi.org/10.1016/j.edurev.2009.12.002

Engeström, Y., \& Sannino, A. (2016). Expansive learning on the move: insights from ongoing research/El aprendizaje expansivo en movimiento: aportaciones de la investigación en $\begin{array}{lllll}\text { curso. } & \text { Infancia } & y & \text { aprendizaje, } & 39(3),\end{array}$ https://www.doi.org/10.1080/02103702.2016.1189119

Etikan, I., Musa, S. A., \& Alkassim, R. S. (2016). Comparison of convenience sampling and purposive sampling. American Journal of Theoretical and Applied Statistics, 5(1), 1-4. https://www.doi.org/10.11648/j.ajtas.20160501.11

Fazio, M. E. (2009). Pragmática y argumentación en el discurso publicitario. El caso de la campaña de SanCor Bio en Argentina. Pensar la Publicidad. Revista Internacional de Investigaciones Publicitarias, 2(2),

15-36. https://revistas.ucm.es/index.php/PEPU/article/view/PEPU0808220015A

Firestone, R., Rowe, C. J., Modi, S. N., \& Sievers, D. (2017). The effectiveness of social marketing in global health: a systematic review. Health policy and planning, 32(1), 110-124. https://doi.org/10.1093/heapol/czw088

Friedman, A. L., Kachur, R. E., Noar, S. M., \& McFarlane, M. (2016). Health communication and social marketing campaigns for sexually transmitted disease prevention and control: What 
is the evidence of their effectiveness? Sexually transmitted diseases, 43(2S), S83-S101. https://doi.org/10.1097/OLQ.0000000000000286

González Palacio, E. V., Castellano Ascencio, M. D., y Sepúlveda Tamayo, N. A. (enero-abril, 2021). Diseño y validación de un cuestionario sobre literacidad en educación superior. Revista Virtual Universidad Católica del Norte, (62), 63-87. https://www.doi.org/10.35575/rvucn.n62a4

González, E. R., Esteban, M. P. P., y Luis, H. G. (2016). Análisis del modelo de gestión de la comunicación y las RRPP de las ONG en Valencia en una sociedad en crisis (2012). ZERRevista de Estudios de Comunicación, 21(40), 85-118. https://doi.org/10.1387/zer.15513

González-Cabrera, C. (2017). El publicista social en la convergencia del Buen Vivir y los ODS. Chasqui. Revista Latinoamericana de Comunicación, (134), 143-159. https://dialnet.unirioja.es/servlet/articulo?codigo=5974559

Göttlichová, M., \& Soukalová, R. (2015). Options for innovation of marketing approaches to the market in the non-profit sector. Procedia-Social and Behavioral Sciences, 175, 334-341. https://doi.org/10.1016/j.sbspro.2015.01.1208

Gumucio-Dagron, A. (2011). Comunicación para el cambio social: clave del desarrollo $\begin{array}{llll}\text { participativo. Signo } & y & \text { Pensamiento, 30(58), }\end{array}$ https://www.redalyc.org/pdf/860/86020038002.pdf

Gutiérrez Guerrero, M. D., y Moscardó Herrera, C. (2012). La Nominalización en la Redacción Publicitaria. Análisis de la ausencia de la marca en el eslogan. En Actas IV Congreso Internacional Latina de Comunicación Social: Comunicación, control y resistencias (p. 125). Sociedad Latina de Comunicación Social. http://www.revistalatinacs.org/12SLCS/2012_actas/100_Gutierrez.pdf

Huertas-García, R., Gázquez-Abad, J. C., y Lengler, J. (2014). Publicidad conjunta marca-causa: el papel de la implicación del individuo hacia la publicidad en la comprensión y evaluación del mensaje. Revista Europea de Dirección y Economía de la Empresa, 23(3), 147-155. https://doi.org/10.1016/j.redee.2014.03.003

Hernández, A. E. (2010). Usos de la literatura en la publicidad. Pensar la Publicidad, 4(2), 141167. https://revistas.ucm.es/index.php/PEPU/article/view/PEPU1010220141A/15048 
i Suñé, D. B., y Foix, N. A. (2011). ¿Traducción o adaptación? Fórmulas para la creación de cuñas y spots en una campaña publicitaria. Vivat Academia, 429-442. https://doi.org/10.15178/va.2011.117E.429-442

Islas Torres, C., y Carranza Alcántar, M. D. R. (septiembre-diciembre, 2020). Análisis de contenido de una experiencia formativa a través de aula invertida. Revista Virtual Universidad Católica del Norte, (61), 3-18. https://www.doi.org/10.35575/rvucn.n61a2

Kain, D., \& Wardle, E. (2014). Activity theory: An introduction for the writing classroom. Wardle and Downs, 273-283. https://tinyurl.com/46ejqlpt

Kubacki, K., \& Szablewska, N. (2019). Social marketing targeting Indigenous peoples: a systematic review. Health Promotion International, 34(1), 133-143. https://doi.org/10.1093/heapro/dax060

Lahoz, J. V. (2007). Estrategias lingüísticas de los textos publicitarios (Vol. 92). Editorial UOC.

Liston-Heyes, C., \& Liu, G. (2013). A study of non-profit organisations in cause-related marketing: Stakeholder concerns and safeguarding strategies. European Journal of Marketing, 47(11/12), 1954-1974. https://doi.org/10.1108/EJM-03-2012-0142

López, M. A. (2009). ¿Publicidad social? Usos y abusos de "lo social” en la publicidad. ICONO 14, 7(2), 125-151. https://www.redalyc.org/pdf/5525/552556589008.pdf

Merriam, S. B. (1998). Qualitative Research and Case Study Applications in Education. Revised and Expanded from" Case Study Research in Education". Jossey-Bass Publishers.

Navarro, F., Ávila Reyes, N., Tapia-Ladino, M., Cristovão, V. L., Moritz, M. E. W., Narváez Cardona, E., y Bazerman, C. (2016). Panorama histórico y contrastivo de los estudios sobre lectura y escritura en educación superior publicados en América Latina. Revista Signos, 49, 78-99. http://dx.doi.org/10.4067/S0718-09342016000400006

Nieto, R. M. (2007). La comunicación web de las ONGD. Signo y Pensamiento, 26(51), 130-136. https://www.redalyc.org/pdf/860/86005112.pdf

Parodi, G., y Burdiles, G. (2015). Leer y escribir en contextos académicos y profesionales: géneros, corpus y métodos. Ariel.

Pawlak, A. (2020). Whose education is it? Social consequences of commercial advertising campaigns. Problemy Opiekuńczo-Wychowawcze, 592, 69. https://www.doi.org/10.5604/01.3001.0014.3570 
Prior, P., \& Shipka, J. (2003). Chronotopic lamination: Tracing the contours of literate activity. Writing Selves, Writing Societies: Research from activity perspectives, 180-238. https://tinyurl.com/5f9tc2w2

Rey, J. (2011). Evolución de la redacción publicitaria en el siglo XX: estudio sobre la longitud de la frase y la extensión del cuerpo de texto en la publicidad en castellano. Trípodos, (27), 141-158. http://hdl.handle.net/11441/61329

Riaño, V. S. (2016). Sobre la constitución del acto discursivo en el mensaje publicitario. Presupuestos filosóficos desde el pragmatismo. $V$ Encuentro Internacional de Investigadores en Publicidad, 129-142. https://tinyurl.com/11hbjg3r

Rounsaville, A. (2012). Selecting Genres for Transfer: The Role of Uptake in Students' Antecedent Genre Knowledge. $\quad$ Composition $\quad$ Forum (26), 1-16. https://files.eric.ed.gov/fulltext/EJ985811.pdf

Russell, D. R. (2007). Rethinking the articulation between business and technical communication and writing in the disciplines: Useful avenues for teaching and research. Journal of Business and Technical Communication, 21(3), 248-277. https://doi.org/10.1177/1050651907300452

Russell, D. R., \& Cortes, V. (2012). Academic and scientific texts: The same or different communities? In Monserrát Castelló \& Christine Donahue (Eds.), University writing: Selves and Texts in Academic Societies (pp. 3-18). Brill. https://lib.dr.iastate.edu/cgi/viewcontent.cgi?article=1278\&context=engl_pubs

Schettini, P., y Cortazzo, I. (2015). Análisis de datos cualitativos en la investigación social. Editorial de la Universidad Nacional de La Plata (EDULP). http://sedici.unlp.edu.ar/bitstream/handle/10915/49017/Documento_completo.pdf?sequen ce $=1 \&$ is Allowed $=y$

Senes, F., y Ricciulli-Duarte, D. (2019). La publicidad social como herramienta para la construcción de ciudadanía. Razón Crítica, 6, 183-199. https://doi.org/10.21789/25007807.1425

Spinuzzi, C. (2014). How nonemployer firms stage-manage ad hoc collaboration: An activity theory analysis. Technical Communication Quarterly, 23(2), 88-114. https://doi.org/10.1080/10572252.2013.797334 
Spinuzzi, C. (2015). Toward a typology of activities: Understanding internal contradictions in multiperspectival activities. Journal of Business and Technical Communication, 29(1), 335. https://doi.org/10.1177/1050651914548277

Toca Torres, C. E. (2013). El marketing al servicio de las organizaciones del tercer sector. Estudios Gerenciales, 29(129), 386-395. https://doi.org/10.1016/j.estger.2013.11.004

Toledo, P. F. (2007). El papel de la fraseología en el discurso publicitario: sugerencias para un análisis multidisciplinar/The role of phraseology in the language of advertising. Pensar la Publicidad, 1(1), 181-198. https://core.ac.uk/download/pdf/38818501.pdf

Tuomi-Gröhn, T., Engeström, Y., \& Young, M. (2003). From transfer to boundary-crossing between school and work as a tool for developing vocational education: An introduction. In T. Tuomi-Gröhn, \& Y. Engeström (Eds.), Between school and work: New perspectives on transfer and boundary-crossing. Pergamon Press.

Wenger, E. (2010). Communities of practice and social learning systems: the career of a concept. In Chris Blackmore (Eds.), Social learning systems and communities of practice (pp. 179198). Springer. https://doi.org/10.1007/978-1-84996-133-2_11

Winsor, D. A. (2013). Writing like an engineer: A rhetorical education. Routledge. 


\section{Anexo 1}

Protocolo de entrevista

Publicidad y organizaciones no lucrativas (ONG) en el Valle del Cauca: comprendiendo el rol de la comunicación y la escritura en la publicidad social

\section{Objetivos de la entrevista:}

- Describir ciclos de flujo y producción de contenidos en los que se involucra un publicista social en las ONG.

- Describir la escritura en publicidad social desarrollada por las ONG en el Valle del Cauca.

[Inicio, saludo y agradecimiento/Recordar el objetivo de la investigación/Suministrar el consentimiento]

\section{Información de la organización}

1. Describa la organización en que trabaja

[Nos puedes compartir información de la organización en la cual trabajas (pequeña historia, misión, visión, organigrama) material físico preferiblemente]

\section{Perfil y trayectoria del profesional}

2. ¿Cuánto tiempo lleva laborando en la organización?

3. ¿En qué otras organizaciones ha trabajado?

4. ¿Cuáles fueron sus funciones en dichas organizaciones?

5. ¿Qué tipo de problemas profesionales resolvía en dichas organizaciones?

6. ¿Qué cargo que desempeña actualmente y cuáles son sus funciones?

7. Describa el tipo de actividades profesionales que usted resuelve en esta organización

8. Por favor, cuéntenos cómo terminó trabajando en una organización como esta

\section{Descripción de un proyecto profesional desafiante}

Para explorar sus experiencias profesionales de comunicación en su organización, por favor, piense en una experiencia que haya significado un reto profesional en la ONG

9. ¿Cuénteme en qué consistió dicha experiencia, proyectos, trabajo?

10. ¿Por qué lo recuerda de ese modo?

11. ¿Quiénes participan?, ¿qué labores desarrollaron? ¿cuál era el objetivo de esta comunicación? ¿Cuál fue la duración?

12. ¿Cuál era el objetivo/la meta de dicha experiencia, proyectos, trabajo?

13. ¿Cuál era su rol?

14. ¿Cuál era la meta de su trabajo?

15. Liste los productos de comunicación (entregables) relacionados con su rol y su meta

IV. Descripción de la ruta de procedimientos utilizados para producir un entregable desafiante

Por favor, seleccione sólo uno de los entregables que piense fue el más desafiante para producir por usted, y responda las siguientes preguntas. 
16. Por qué lo recuerda de ese modo [Se inicia el dibujo -- incluyendo el escenario sobre cómo se empieza a desarrollar dicho entregable]

17. Describa los recursos/personas con los que contaban para realizarlo -- dónde estaban

18. Explíqueme los procedimientos en orden (o la ruta) de producción del entregable

19. Describa los participantes y sus roles a lo largo de la ruta de producción (grafique mientras habla o después de hablar):

Cuánto es el tiempo de producción

Quién escribe/produce --cuándo--para qué--cómo--con qué tiempos

Quién lee/revisa/propone--cuándo--para qué--cómo-con qué tiempos

20. En qué momento de la ruta se presentan obstáculos, menciones cuáles e ilústrenos con ejemplos

Qué consecuencias traerían errores en la producción de este entregable y para quiénes Qué consecuencias traerían el éxito en la producción de este entregable y para quiénes

\section{Conceptualizar publicidad en organizaciones no lucrativas (ONG)}

21. Para finalizar, si tuviera que darles consejos a futuros profesionales de comunicación sobre cómo prepararse para gestionar la publicidad en ONG, ¿qué les diría? 